\title{
Justizjuristen und NS-Vergangenheit
}

Die Justiz hat mit der Aufarbeitung ihrer Rolle im Nationalsozialismus auch 39 Jahre nach Kriegsende noch erhebliche Probleme. In der Aus- und Fortbildung von Juristen ist das Thema bis heute weitgehend tabu geblieben. Die "Bewältigung " der NS-Zeit wurde vor allem den Historikern überlassen, was der verbreiteten Juristenmeinung Ausdruck gibt, das Versagen der Justiz im Dritten Reich stelle sich als Betriebsunfall der Rechtsgeschichte dar und sei allenfalls als historische Episode von Interesse. Für diese Abwehrhaltung geben Vorgeschichte und Ablauf der ersten Fortbildungstagung zum Thema NS-Justiz an der bereits 10 Jahre bestehenden Deutschen Richterakademie in Trier ein gutes Beispiel.'

Die vom Niedersächsischen Justizministerium ausgerichtete Tagung fand vom I 1 . bis 17 . Dezember 1983 statt und war erst nach langen öffentlichen Diskussionen zustandegekommen. Schon Anfang 1980 forderten die in der Gewerkschaft ÖTV organisierten Richter und Staatsanwälte, an der Deutschen Richterakademie eine Tagung zum Thema NS-Justiz durchzuführen. 198 I von der Programmkonferenz der Richterakademie für Dezember ${ }_{19} 83$ beschlossen, wurde die Tagung Anfang 1982 von Niedersachsen wieder abgesagt, u. a. mit der wenig überzeugenden Begründung, dieses schwierige Thema bedürfe wegen der zu erwartenden Öffentlichkeitswirkung sorgfältiger Vorbereitung, und dafür reiche die verbleibende Zeit ( $1 / 2$ Jahre!) nicht aus; im übrigen gebe es für die Richterfortbildung wichtigere Themen. Dies stieß auf den massiven Protest der Richter und Staatsanwälte in der ÖTV, der in der Öffentlichkeit insbesondere in der überregionalen Presse große Resonanz fand. Der damalige Justizminister Schmude kritisierte ebenfalls die Haltung des Niedersächsischen Justizministeriums und erklärte sich - wie auch einige Länderjustizminister - bereit, notfalls selbst die Tagung zu übernehmen. Schließlich sah sich der niedersächsische Justizminister Remmers im August 1982 doch in der Lage, die abgesagte Tagung wieder in das Programm der Richterakademie aufzunehmen.

Wurden damit die "Berührungsängste " gegenüber der Thematik deutlich, so ließ die Formulierung des Tagungsthemas »Recht im Nationalsozialismus « und nicht "Justiz im Nationalsozialismus « befürchten, daß es nicht um eine vorbehaltlose Aufarbeitung des Versagens der im Nationalsozialismus tätigen Richter und Staatsanwälte gehen sollte. Eine Befürchtung, die sich jedenfalls hinsichtlich eines Teils der von Justizpraktikern vorgetragenen Referate als berechtigt erwiesen hat. Denn neben wissenschaftlich ausgewiesenen Fachleuten standen auf der Referentenliste auch Repräsentanten der - vornehmlich niedersächsischen - Justiz, deren Einladung sich eher mit ihren hohen Ämtern als mit ihrer besonderen Sachkompetenz erklären läßt.

I Eine ausführliche Darstellung der Tagung hat der Verfasser in der Zeitschrift des DGB »Der Deutsche Beamte $1984 /$ Heft 3 , S. $\varsigma_{1}$ ff. veröffentlicht. 
Andererseits wurde gerade durch diese Referenten den Tagungsteilnehmern und den anwesenden zahlreichen Journalisten (das starke Medieninteresse war sicherlich ein Novum an der Deutschen Richterakademie) eindrucksvoll vor Augen geführt, wie ein Großteil der Justizjuristen heute noch über die NS-Vergangenheit denkt.

Für den ersten Eklat sorgte der Leitende Senatsrat Gerhard Spletzer aus Berlin, der zum Thema "Der Volksgerichtshof, sein Wirken aus damaliger und heutiger Sicht" sprach. Spletzer war Ende der 6oer Jahre selbst als Staatsanwalt für die Verfolgung der ehemaligen Richter des Volksgerichtshofs zuständig und führt heute die Dienstaufsicht über die gegen die jetzt noch 5 I Beschuldigten ermittelnden Staatsanwälte. Der Referent setzte sich u. a. damit auseinander, ob der Volksgerichtshof ein ordentliches Gericht im Sinne des $\$$ I GVG war und ob den Richtern Rechtsbeugung vorgeworfen werden kann. Spletzer führte dann sinngemäß aus, diese Fragen seien nicht einfach zu beantworten und bedürften sorgfältiger Prüfung anhand jedes einzelnen Volksgerichtshofurteils. Er habe z. B. Todesurteile des Volksgerichtshofs wegen Hoch- und Landesverrats gelesen, die rechtlich nicht $\mathrm{zu}$ beanstanden seien und die er heute - wenn er Richter wäre - nicht anders fällen würde (!). Trotz des heftigen Protests von Teilnehmern, die ihre Betroffenheit über diese Entgleisung des Referenten zum Ausdruck brachten und die darauf hinwiesen, daß der Vorwurf des Hoch- und Landesverrats auf dem Hintergrund des NS-Unrechtsregimes gesehen werden müsse und im übrigen die Todesstrafe keineswegs zwingend verhängt werden mußte, blieb Spletzer bei der Äußerung. Er machte damit in erschreckender Weise eine Juristenmentalität deutlich, die auch heute noch verbreitet ist und die in dem bekannten Filbinger-Satz Ausdruck findet: »Was damals Recht war, kann heute nicht Unrecht sein «.

Für den zweiten Eklat sorgte der Präsident des Oberlandesgerichts Celle, Dr. Harald Franzki, der das Thema »Die Bewältigung der Vergangenheit - NSVerbrechen und ihre strafrechtliche Ahndung nach dem Kriege « übernommen hatte. Der Referent fügte der ausführlichen Darstellung der NS-Greueltaten (Franzki ging offenbar davon aus, daß seine Zuhörer insoweit keine Vorkenntnisse hatten) persönliche Anmerkungen hinzu, die im Zuhörerkreis den Eindruck der Verharmlosung erweckten und eine Tendenz zur Aufrechnung erkennen ließen. Zum Beispiel erklärte der Referent, die große Zahl der zu Tode gekommenen sowjetischen Kriegsgefangenen u. a. damit, daß die Soldaten bereits verwundet und unterernährt in deutsche Kriegsgefangenschaft geraten und angesichts der Versorgungsprobleme nicht mehr zu retten gewesen seien. Bei seinen rechnerischen Überlegungen zur Gesamtzahl der ermordeten Juden wies Franzki einschränkend darauf hin, daß beispielsweise die von dem Lagerkommandanten Höss für Auschwitz genannten Zahlen aus Prahlsucht zu hoch gegriffen waren. Als der Referent nach weiteren Anmerkungen dieser Art schließlich von der »Nürnberger Siegerjustiz « sprach und in diesem Zusammenhang die Tatsache für erwähnenswert hielt, daß die Ermordung polnischer Offiziere in Katyn nicht ein Werk der Nazis, sondern der Sowjets gewesen sei, verließ der polnische Professor Pilichowski, Leiter der Warschauer Hauptkommission zur Verfolgung von NS-Verbrechen und Gast der Tagung, unter Protest den Saal. In der nachfolgenden Diskussion stießen die Ausführungen Franzkis auf erheblichen Widerspruch. Ein Richter bezeichnete die Äußerungen zu Recht als unterschwellige Diminuierung deutscher Schuld.

Derartige Kritik wurde allerdings nur von einer Minderheit der ca. 45 Tagungsteilnehmer geübt. Bezeichnenderweise beteiligten sich daran so gut wie gar nicht die anwesenden höherrangigen Justizvertreter, wie Bundesrichter, Vorsitzende Richter, General- und Oberstaatsanwälte, die immerhin etwa die Hälfte der Teilnehmer stellten. 
Im übrigen war aus Diskussionsbeiträgen auch der jüngeren Richter und Staatsanwälte zu entnehmen, daß die seit 1945 immer wieder zu hörenden Begründungsideologien noch weit verbreitet sind. So wurde mehrfach die Auffassung vertreten, der rechtstheoretische Positivismus habe die Richter wehrlos dem NS-System ausgeliefert, und die Justiz sei einer starken politischen Steuerung unterworfen gewesen. Diese Behauptungen wurden aber in den fundierten Referaten von Gotthard Jasper, Diemut Majer und Bernd Rüthers mit eindrucksvollen Beispielen widerlegt.

Eine gefährliche Neigung zur Relativierung des Anteils der Justiz an der NaziDiktatur war auch herauszuhören, wenn Teilnehmer betonten, daß keineswegs die gesamte Justiz willfährig gewesen sei und es Beispiele mutiger Entscheidungen gegeben habe; sogar vom Volksgerichtshof sei öfter auf Freispruch erkannt worden - als ob diese Einzelbeispiele das Gewicht der terroristischen Kernfunktion der Justiz verändern könnten. Hingewiesen wurde auch darauf, daß Terrorurteile in der Strafjustiz oftmals dem negativen Einfluß ehrenamtlicher Richter zuzuschreiben gewesen seien.

Bezeichnend war zudem, daß über mögliche Konsequenzen aus der NS-Zeit für die heutige Justiz und über das frühzeitige Erkennen ähnlicher Entwicklungen kaum gesprochen wurde. Vielmehr entstand ein Sturm der Entrüstung, als von einem Teilnehmer im Zusammenhang mit den nationalsozialistischen Bestrebungen zur Änderung des Strafverfahrensrechts gewisse inhaltliche Parallelen zu den in der sogenannten "Horrorliste « zusammengefaßten "Reform-Vorstellungen « der Länderjustizminister aufgezeigt wurden. Das galt als unpassend.

Solange Richter und Staatsanwälte heute immer noch - sei es aus Überzeugung oder aus Ignoranz - die angebotenen Begründungsideologien für das Versagen der Justiz in der Weimarer Zeit und im Nationalsozialismus übernehmen und nicht zu einer vorbehaltlosen Aufarbeitung und Ursachenerforschung bereit sind, solange werden sie nicht in der Lage sein, ähnliche politische Entwicklungen zu erkennen und ihnen zu widerstehen. Als ersten Schritt dahin müssen die deutschen Juristen lernen, ihre »organisierte Unbußfertigkeit« (Iwand) zu überwinden. Die Tagung in Trier läßt insoweit allerdings keine übermäßigen Hoffnungen zu.

Bernd Asbrock 